\title{
Características adaptativas da associação simbiótica e da fixação biológica do nitrogênio molecular em plantas jovens de Lonchocarpus muehlbergianus Hassl., uma leguminosa arbórea nativa do Cerrado
}

\author{
Adaptive characteristics of the symbiotic association and nitrogen symbiotic fixation in young \\ plants of Lonchocarpus muehlbergianus Haasl., a leguminous tree native from 'Cerrado'
}

\author{
Vitor Moreira ${ }^{1}$, Gilberto Costa Justino ${ }^{2}$, Liliane Santos Camargos $^{3,4}$ \& Leandro Ferreira Aguiar ${ }^{1}$
}

\begin{abstract}
Resumo
O nitrogênio mineral afeta negativamente a simbiose e a fixação biológica do nitrogênio em plantas cultivadas. Entretanto, este efeito não é verificado em algumas espécies arbóreas, pouco estudadas até o momento. O objetivo deste trabalho foi avaliar a capacidade de Lonchocarpus muehlbergianus, espécie arbórea nativa do Cerrado, em utilizar o nitrogênio molecular, na presença do nitrogênio mineral (nitrato), característica desejável para plantas fixadoras crescendo em solos com nitrato como principal forma de nitrogênio disponível. Foram determinados o número e a massa seca dos nódulos, da parte aérea e das raízes, bem como o conteúdo de nitrato, aminoácidos e ureídeos, transportados pelo xilema e a atividade da redutase do nitrato. Foi observado que o número e a massa seca dos nódulos não foram negativamente afetados pelo nitrato. Ocorreu pequeno aumento na massa seca da parte aérea e do sistema radicular de plantas noduladas tratadas com nitrato, resultado verificado também para os teores de aminoácidos, ureídeos e atividade da redutase do nitrato. Os resultados obtidos sugerem que a espécie tem capacidade de utilizar tanto nitrogênio mineral quanto molecular, uma vez que o transporte de ureídeos, do sistema radicular para a parte aérea, não foi reduzido em plantas noduladas tratadas com nitrato.

Palavras-chave: crescimento de plantas, estresse em plantas, nodulação, transporte pelo xilema, tolerância de plantas a estresses abióticos.
\end{abstract}

\begin{abstract}
It is well established that mineral nitrogen negatively affects symbioses and molecular nitrogen utilization by crops. Nevertheless, symbiosis and nitrogen fixation of tree species, less studied, has been showed certain tolerance to nitrate. The aim of this work was to evaluated the nitrogen molecular use by nodulated Lonchocarpus muehlbergianus, a Cerrado legume tree, even supplied with mineral nitrogen as nitrate, important feature for symbiotic plants growing in soil where nitrate is the prominent source of nitrogen. For this purpose, the number and dry weight of nodules, shoot and roots, as well as content of xylem sap amino acids, nitrate and ureides, product of symbiotic nitrogen fixation, and nitrate reductase activity were determined. At the end of the experiment, it was observed that the number and dry weight of nodules was not affected by nitrate. Addictionally, it was verified a short increment in shoots and roots treated with nitrate. Similar results were found for amino acids and ureides content and foliar nitrate reductase activity. Our results suggest that Lonchocarpus muehlbergianus is able to utilize at the same time both mineral and molecular nitrogen since transport of ureides from roots to shoots was not reduced when nodulated plants was fed with nitrate.
\end{abstract}

Key words: plant growth, plant stress, nodulation, xylem transport, plant tolerance to abiotic stress.

\section{Introdução}

Lonchocarpus muehlbergianus Hassl (feijãocru) é uma leguminosa arbórea heliófita, decídua, encontrada em florestas semidecíduas da Bacia do
Rio Paraná, com características ornamentais devido à beleza de suas flores, e de acordo com Lorenzi (2000) pode ser uma alternativa em plantios mistos que visam à recuperação de áreas degradadas. Neste

\footnotetext{
'Universidade Federal do Mato Grosso do Sul, Campus de Três Lagoas, Depto. Ciências Naturais, 79601-100, MS, Brasil.

${ }^{2}$ Universidade Federal de Alagoas, Inst. Ciências Biológicas e da Saúde, 57010-020, AL, Brasil.

${ }^{3}$ UNESP - Universidade Estadual Paulista, Faculdade de Engenharia de Ilha Solteira, Depto. Biologia e Zootecnia, 15385-000, SP, Brasil.

${ }^{4}$ Autor para correspondência: camargos@bio.feis.unesp.br
} 
contexto, a espécie tem grande potencial para o uso em recomposição de matas, especialmente, em áreas ripárias da Mata Atlântica onde ocorreu construção de hidroelétricas. No que diz respeito ao estágio de sucessão ecológica, a espécie foi classificada como pioneira (Cancian 1999) ou como secundária inicial (Instituto Florestal de São Paulo 2003). O potencial de recuperação de áreas degradadas da espécie está relacionado à capacidade de associação a bactérias fixadoras de nitrogênio, genericamente conhecidas como rizóbios, aspecto importante para a recuperação das características físicas e químicas do solo (Nichols \& Carpenter 2006).

Em sistemas agroflorestais, a associação de leguminosas com os rizóbios é a principal responsável pela entrada de $\mathrm{N}$ combinado no ambiente. Neste âmbito, a associação simbiótica entre bactérias e plantas, bem como, a fixação biológica do nitrogênio (FBN) tem características distintas que interferem no potencial de utilização do nitrogênio molecular $\left(\mathrm{N}_{2}\right)$ em diferentes escalas. Sendo assim, Herridge \& Rose (2000) postularam que o melhoramento genético de plantas de soja (Glycine max), visando a obtenção de adequada associação simbiótica e FBN, deveria estar ligado a linhas de pesquisa que contemplassem: (1) a tolerância da simbiose ao nitrato $\left(\mathrm{NO}_{3}^{-}\right)$, (2) a mutagênese induzindo supernodulação e (3) a seleção de cultivares com nodulação específica ou promíscua, dependendo das circunstâncias. Estas são características que apresentam variabilidade genética e que permitem a seleção de associações mais eficientes no processo de FBN.

A tolerância da $\mathrm{FBN}$ ao $\mathrm{NO}_{3}{ }^{-}$presente no meio do cultivo foi verificada em algumas linhagens de plantas de soja (Betts \& Herridge 1987). Este aspecto é importante, pois a tolerância ao $\mathrm{NO}_{3}^{-}$aumenta a porcentagem de $\mathrm{N}$ na biomassa proveniente da FBN, o que potencializa a contribuição das leguminosas para a recuperação das condições de fertilidade do solo, elevando a disponibilidade de $\mathrm{N}$ para plantas associadas em condições naturais, disponibilizando $\mathrm{NO}_{3}{ }^{-}$para espécies não fixadoras (Omena-Garcia et al. 2011).

A variabilidade para tolerância ao $\mathrm{NO}_{3}{ }^{-}$existe dentro de populações naturais podendo ser induzida por mutagênese (Jacobsen \& Feenstra 1984; Carroll et al. 1985a,b; Betts \& Herridge 1987; Herridge \& Betts 1985, 1988; Park \& Buttery 1988; Hardarson \& Atkins 2003) e utilizada para o melhoramento genético (Park \& Buttery 1989; Lambe et al.
2008). Forrester et al. (2006) revisaram os artigos publicados sobre a produtividade de Eucaliptus globulus com espécies arbóreas fixadoras de nitrogênio e verificaram efeito positivo na produtividade primária em todos os casos. $\mathrm{O}$ aumento da tolerância ao $\mathrm{NO}_{3}{ }^{-}$, relacionado à habilidade da leguminosa nodular e fixar nitrogênio na presença de $\mathrm{NO}_{3}{ }^{-}$no solo, pode não resultar em aumento da produtividade de biomassa ou grãos do indivíduo, mas aumenta a porcentagem de nitrogênio do indivíduo derivado da fixação aumentando benefícios residuais à fertilidade do solo (Herridge \& Rose 2000). Desta maneira, a tolerância ao $\mathrm{NO}_{3}{ }^{-}$aumenta a produtividade de ecossistemas quando utilizadas em consórcio ou para culturas que a sucedem, fornecendo nitrogênio fixado ao sistema sem que ocorra concorrência por este elemento.

De acordo com Glyan'ko et al. (2009), em condições de alta concentração de nitrogênio mineral no ambiente, a planta passa a reconhecer o Rhizobium como um patógeno. Mas, espécies e cultivares que mantêm a fixação na presença de 5 mM de $\mathrm{NO}_{3}{ }^{-}$são consideradas altamente tolerantes. As informações existentes sobre tolerância de plantas noduladas ao $\mathrm{NO}_{3}{ }^{-}$restringem-se a espécies anuais de leguminosas cultivadas, sendo encontrados poucos trabalhos sobre a avaliação da tolerância da fixação biológica ao $\mathrm{NO}_{3}{ }^{-}$em espécies arbóreas (Omena-Garcia et al. 2011). Entretanto, as respostas da associação simbiótica e da fixação biológica das espécies estudadas até agora foram bastante diversas, algumas sendo fortemente inibidas pela presença do nitrogênio mineral em ambas as formas, tanto $\mathrm{NO}_{3}^{-}$quanto amônio $\left(\mathrm{NH}_{4}{ }^{+}\right)$e em outras resistindo a concentrações consideravelmente altas deste elemento ou mesmo induzindo a simbiose, produzindo maior número e biomassa de nódulos (Dan \& Brix 2009; Camargos \& Sodek 2010; Omena-Garcia et al. 2011).

As alterações provocadas pelos fatores ambientais à FBN podem ser avaliadas a partir da determinação de compostos nitrogenados transportados do sistema radicular para a parte aérea através do xilema (Justino \& Sodek 2013). O transporte de ureídeos, produtos diretos da FBN, pode ser utilizado como meio de avaliação da eficiência da atividade dos nódulos em leguminosas transportadoras de ureídeos (Amarante et al. 2006; Camargos \& Sodek 2010). Esta característica também pode ser analisada pelo perfil de aminoácidos (Amarante \& Sodek 2006). 
Em alguns casos, tem sido observada a redução no transporte de ureídeos em função da presença do nitrogênio mineral e de outros fatores de estresses como o alagamento, o que indicou redução das taxas de utilização do nitrogênio molecular (Sodek \& Silva 1996; Amarante et al. 2006).

No presente trabalho avaliamos a eficiência da FBN a partir da determinação de ureídeos na seiva do xilema, com o objetivo de verificar a capacidade de Lonchocarpus muehlbergianus em fixar $\mathrm{N}_{2}$ mesmo na presença do nitrogênio mineral, na forma de $\mathrm{NO}_{3}^{-}$, uma das formas reduzidas encontradas em maiores concentrações em solos.

\section{Material e Métodos}

\section{Obtenção e cultivo das plantas}

Plantas jovens de Lonchocarpus muehlbergianus com aproximadamente quatro meses de idade foram fornecidas pela Divisão de Implantação e Manutenção de Programas Ambientais da Companhia Energética do Estado de São Paulo (CESP). As plantas cultivadas em tubetes foram transferidas para vasos com capacidade para $3 \mathrm{~L}$ contendo vermiculita como substrato e, imediatamente, inoculadas com macerado obtido de nódulos coletados de plantas da mesma espécie. Após o estabelecimento da nodulação, as plantas passaram a receber solução nutritiva de Hoagland (Hoagland \& Arnon 1938), com ou sem nitrogênio, $100 \mathrm{~mL}$ por vaso, duas vezes por semana durante vinte dias. Os tratamentos foram: solução sem nitrogênio (T1), solução com 7,5 mM de $\mathrm{NO}_{3}{ }^{-}$(T2) e solução com 22,5 mM de $\mathrm{NO}_{3}^{-}$(T3).

\section{Coleta do material vegetal}

Após 20 dias de fornecimento de nitrato às plantas, a seiva do xilema foi coletada com a utilização de uma bomba de pressão baseado no descrito por Scholander et al. (1965). Adicionalmente, folhas, raízes e nódulos foram coletados. Parte do material foi utilizada para a obtenção do peso da massa seca e outra parte para a extração e determinação de compostos nitrogenados.

\section{Extração e determinação}

dos compostos nitrogenados

Ao final do experimento, 20 dias após o início dos tratamentos, folhas, raízes e nódulos foram separados, lavados em água destilada e o peso da massa seca foi determinado depois de 72 horas em temperatura de $70^{\circ} \mathrm{C}$, em estufa de circulação forçada. Aminoácidos, ureídeos e nitrato foram extraídos após a homogeneização de $1 \mathrm{~g}$ de tecido fresco em $10 \mathrm{~mL}$ de MCA (metanol/ clorofórmio/água - 12/5/3 - v/v) de acordo com Bieleski \& Turner (1966). A fase aquosa contendo os compostos nitrogenados solúveis foi recuperada depois da separação de fases iniciada após a adição de clorofórmio e água na proporção de 1:1,5 para cada 4 volumes do sobrenadante. A fase aquosa foi reduzida para volume conhecido por evaporação a $38^{\circ} \mathrm{C}$ e mantida em freezer até análises posteriores.

Os aminoácidos totais foram determinados pelo método descrito por Yemm \& Cocking (1955), adicionando-se às amostras tampão citrato $(0,2$ $\mathrm{M}, \mathrm{pH} 5,0)$, ninidrina ( $5 \%$ ), cianeto de potássio $(0,002 \mathrm{M})$ e etanol $(60 \%)$; a reação foi analisada em espectrofotômetro a $570 \mathrm{~nm}$ e os resultados comparados com curva padrão de leucina $(0,2-0,6$ $\mu$ moles $(\mathrm{mL})$. Os ureídeos foram determinados pelo método descrito por Vogels \& Van Der Drift (1970). Para isto, foi adicionado hidróxido de sódio $(0,5 \mathrm{~N})$ às amostras que permaneceram em banho-maria a $100^{\circ} \mathrm{C}$ por 4 minutos. Em seguida, o ácido clorídrico $0,65 \mathrm{~N}$ foi adicionado às amostras que foram, novamente, mantidas por 4 minutos a $100^{\circ} \mathrm{C}$. Finalmente, os ureídeos foram quantificados após a adição de tampão fosfato, fenilhidrazina e ferricianeto de potássio às amostras; a reação foi analisada em espectrofotômetro a $535 \mathrm{~nm}$ e os resultados comparados com curva padrão de alantoína (12,5-125 ๆmoles $\mathrm{mL})$. $\mathrm{O} \mathrm{NO}_{3}{ }^{-}$foi determinado pelo método do ácido salicílico, após a adição de ácido salicílico e hidróxido de sódio às amostras (Unkovich et al. 2008); a reação foi analisada em espectrofotômetro a 410 nm e os resultados comparados com curva padrão de nitrato $(0,4-4,0 \mu$ moles $/ \mathrm{mL})$. A atividade da enzima redutase do nitrato (NRA) foi determinada de acordo com método de Radin (1974). Para isto foram adicionados $10 \mathrm{~mL}$ de tampão fosfato $0,1 \mathrm{M}$, contendo $100 \mathrm{mM}$ de nitrato, em $\mathrm{pH} 7,5$, a um grama de material fresco (discos foliares e raízes). Para que ocorresse a infiltração do tampão, as amostras foram submetidas a vácuo e mantidas em temperatura de $30^{\circ} \mathrm{C}$ por 30 minutos no escuro. Posteriormente, foram adicionados 1 $\mathrm{mL}$ de de sulfanilamida em $\mathrm{HCl} 1 \%$ e $1 \mathrm{~mL}$ de naftil etilenodiamida $2 \%$ e a leitura realizada em espectrofotômetro em comprimento de onda de 540 $\mathrm{nm}$, e os resultados comparados com curva padrão de nitrito $(1-250 \mu \mathrm{M} / \mathrm{mL})$. 
Delineamento experimental

e análises estatísticas

O delineamento experimental utilizado foi o inteiramente casualizado com três tratamentos: Nutrição sem nitrogênio mineral (T1) e com nitrogênio disponibilizado na forma de nitrato 7,5 $\mathrm{mM}$ (T2) e 22,5 mM (T3) e três repetições, sendo que uma planta por vaso foi considerada para cada parcela. Os dados foram submetidos à análise de variância e as médias comparadas pelo teste de Tukey a 5\% de probabilidade.

\section{Resultados e Discussão}

A maioria das espécies simbióticas fixadoras de nitrogênio estudadas até agora apresentam algum tipo de sensibilidade à presença do nitrogênio mineral, ou seja, reduzem ou mesmo abandonam a fixação biológica do nitrogênio do ar na presença de alguma forma de nitrogênio combinado, como o $\mathrm{NO}_{3}{ }^{-}$e o $\mathrm{NH}_{4}{ }^{+}$. No presente experimento, todas as plantas de L. muehlbergianus nodularam após a inoculação do sistema radicular com o macerado de nódulos obtido de nódulos da mesma espécie (Fig. 1). $\mathrm{O}$ tratamento com $\mathrm{NO}_{3}^{-}$não alterou o peso dos nódulos até o final do experimento (Fig. 2). Os nódulos estavam aparentemente funcionais (dados não mostrados), pois estes apresentaram uma coloração avermelhada, característica da presença de leghemoglobina, proteína necessária ao adequado funcionamento dos nódulos.

Os dados obtidos neste trabalho estão de acordo com a hipótese de que a fixação biológica do nitrogênio nesta espécie não é afetada pelo nitrogênio mineral $\left(\mathrm{NO}_{3}^{-}\right)$presente no meio de cultivo. De fato, a tolerância da associação simbiótica entre plantas e bactérias à presença de $\mathrm{N}$ fixado no solo já foi verificada em condições experimentais (Dakora 1998; Michaelson-Yeates et al. 1998), mas foi sugerido que esta tolerância não deve ocorrer em muitas espécies (Hardarson et al. 1991). Neste sentido, a formação e a manutenção da função dos nódulos na presença do $\mathrm{N}$ mineral não dependem apenas da interação entre a bactéria e a espécie vegetal em questão, pois a tolerância pode variar entre cultivares de uma mesma espécie, como verificado em plantas de soja (Hardarson et al. 1991; Herridge \& Betts 1988; Sodek \& Silva 1996; Herridge \& Rose 2000). Os mecanismos bioquímicos relacionados à fixação biológica do nitrogênio e a utilização do $\mathrm{N}$ mineral são investigados preferencialmente em plantas cultivadas devido a interesses econômicos.
Entretanto, os aspectos bioquímicos que levam a senescência dos nódulos em função da preferência pelo $\mathrm{NO}_{3}{ }^{-}$ainda não são completamente esclarecidos, mas alterações nos níveis de espécies reativas de oxigênio (EROs) já foram verificadas (Groten et al. 2005). Os mecanismos de manutenção da nodulação e da fixação biológica em plantas $L$. muehlbergianus tratadas com $\mathrm{NO}_{3}{ }^{-}$ainda não foram relatados, o que deve fornecer subsídios para a compreensão deste processo de não-abandono da fixação biológica em condições de presença de nitrogênio mineral.

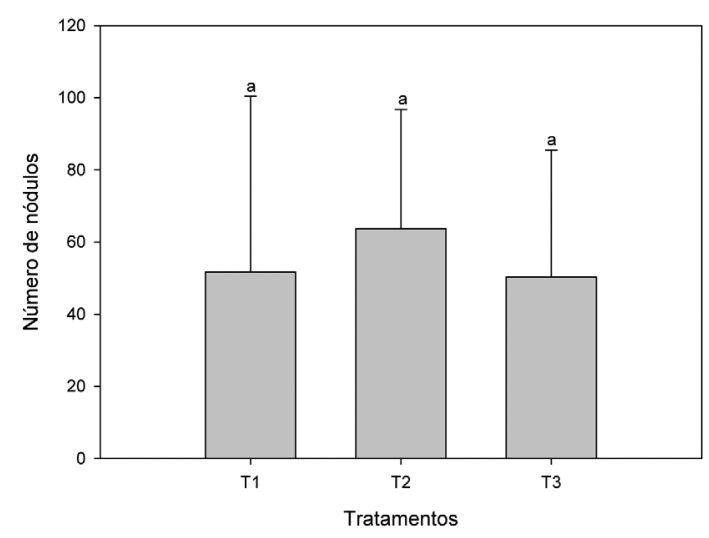

Figura 1 - Número de nódulos em plantas jovens noduladas de L. muehlbergianus tratadas durante 20 dias com nitrogênio mineral na forma de $\mathrm{NO}_{3}^{-}$. T1: sem $\mathrm{N}$; T2: $\mathrm{NO}_{3}^{-} 7,5 \mathrm{mM}$; T3: $\mathrm{NO}_{3}^{-} 22,5 \mathrm{mM}$.

Figure 1 - Nodule number of young nodulated plants of $L$. muehlbergianus treated with mineral nitrogen as $\mathrm{NO}_{3}{ }^{-}$for 20 days. T1: no nitrogen; $\mathrm{T} 2: \mathrm{NO}_{3}^{-} 7,5 \mathrm{mM} ; \mathrm{T} 3: \mathrm{NO}_{3}^{-} 22,5 \mathrm{mM}$.

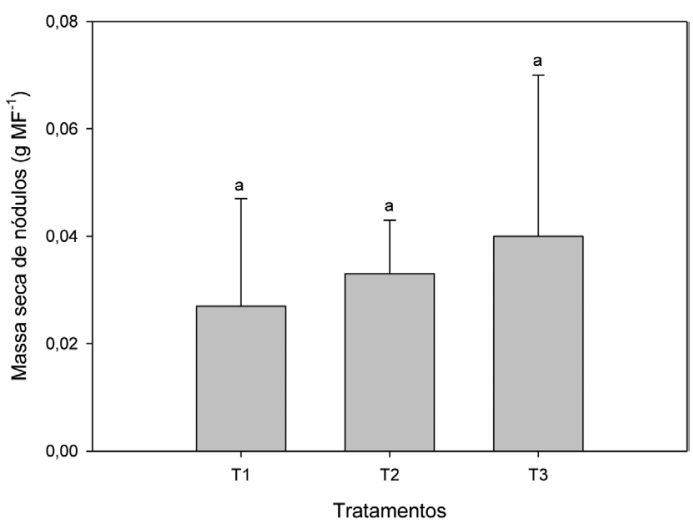

Figura 2 - Massa seca de nódulos de plantas jovens noduladas de L. muehlbergianus tratadas durante 20 dias com nitrogênio mineral na forma de $\mathrm{NO}_{3}{ }^{-}$. T1: sem N; T2: $\mathrm{NO}_{3}^{-}$7,5 mM; T3: $\mathrm{NO}_{3}^{-} 22,5 \mathrm{mM}$.

Figure 2- Nodule dry weight of young nodulated plants of $L$. muehlbergianus treated with mineral nitrogen as $\mathrm{NO}_{3}{ }^{-}$for 20 days. T1: no nitrogen; $\mathrm{T} 2: \mathrm{NO}_{3}^{-} 7,5 \mathrm{mM}$; $\mathrm{T} 3: \mathrm{NO}_{3}{ }^{-} 22,5 \mathrm{mM}$. 
Os efeitos do nitrogênio mineral quanto ao tempo e a forma de aplicação resultam em respostas diferentes entre as espécies vegetais. Exemplo disso é que plantas jovens de Inga edulis (Mart.), leguminosa arbórea nativa da Amazônia, reduziram consideravelmente o número e a massa dos nódulos quando tratadas com $\mathrm{NO}_{3}{ }^{-}(5$ $\mathrm{mM}$ ) em condições controladas (Omena-Garcia et al. 2011). Por outro lado, em Calopogonium mucunoides, uma espécie herbácea nativa do Cerrado, o tratamento com $\mathrm{NO}_{3}^{-}(15 \mathrm{mM})$ causou redução do número, porém não afetou a massa de nódulos das plantas (Camargos \& Sodek 2010). Nesse experimento, a atividade da enzima nitrogenase manteve-se constante quando a planta foi tratada com solução nutritiva contendo $\mathrm{NO}_{3}{ }^{-} 15 \mathrm{mM}$, mas reduziu 59\% quando cultivada em solução com $\mathrm{NO}_{3}{ }^{-} 30 \mathrm{mM}$. Quantidades significativamente menores de $\mathrm{NO}_{3}{ }^{-}$podem causar danos mais drásticos à simbiose em outras espécies, como verificado para Sesbania sesban, em que $\mathrm{NO}_{3}^{-} 2 \mathrm{mM}$ suprimiu completamente a nodulação (Dan \& Brix 2009). Neste aspecto houve grande investimento de biomassa dos nódulos em L. muehlbergianus na presença de nitrato, o que indica que a espécie tem capacidade de utilizar o nitrogênio tanto na forma mineral $\left(\mathrm{NO}_{3}{ }^{-}\right)$quanto na forma molecular $\left(\mathrm{N}_{2}\right)$.

No presente experimento, verificou-se que acúmulo de biomassa total da planta tratada com $\mathrm{NO}_{3}{ }^{-}$não foi significativamente diferente dos demais tratamentos. Embora tenha sido observado discreto aumento (Fig. 3), o N mineral adicional não contribuiu de forma substancial para o crescimento das plantas durante o tempo de execução do experimento. Este resultado foi verificado também quando o acúmulo de massa seca da parte aérea $\mathrm{e}$ das raízes foi analisado (dados não mostrados). Todavia, houve maior investimento em raízes em todos os tratamentos (Fig. 4), característica esta verificada durante o desenvolvimento desta espécie por outros autores (Cancian \& Cordeiro 1998). É possível que em experimentos de longa duração o crescimento de raízes pudesse ser mais pronunciado em relação ao crescimento da parte aérea, caso as condições experimentais fossem mantidas, já que o experimento foi conduzido por 20 dias após o transplantio. Neste caso, a extensão e consequentemente a capacidade de absorção do sistema radicular poderia levar a uma taxa de acúmulo de biomassa superior em plantas tratadas com $\mathrm{N}$ mineral.

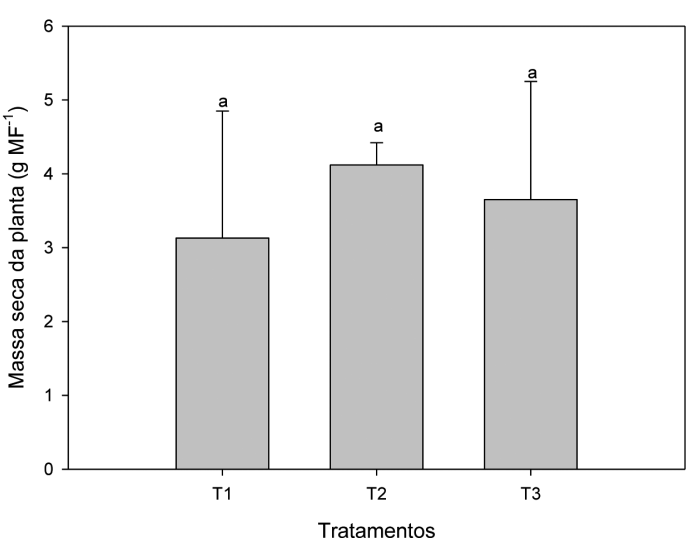

Figura 3 - Peso da massa seca total de plantas jovens noduladas de L. muehlbergianus tratadas durante 20 dias com nitrogênio mineral na forma de $\mathrm{NO}_{3}^{-}$. T1: sem N; T2: $\mathrm{NO}_{3}^{-} 7,5 \mathrm{mM}$; T3: $\mathrm{NO}_{3}^{-} 22,5 \mathrm{mM}$.

Figure 3 - Dry weight of young nodulated plants of $L$. muehlbergianus treated with mineral nitrogen as $\mathrm{NO}_{3}^{-}$for 20 days. T1: no nitrogen; $\mathrm{T} 2: \mathrm{NO}_{3}^{-} 7,5 \mathrm{mM}$; T3: $\mathrm{NO}_{3}^{-} 22,5 \mathrm{mM}$.

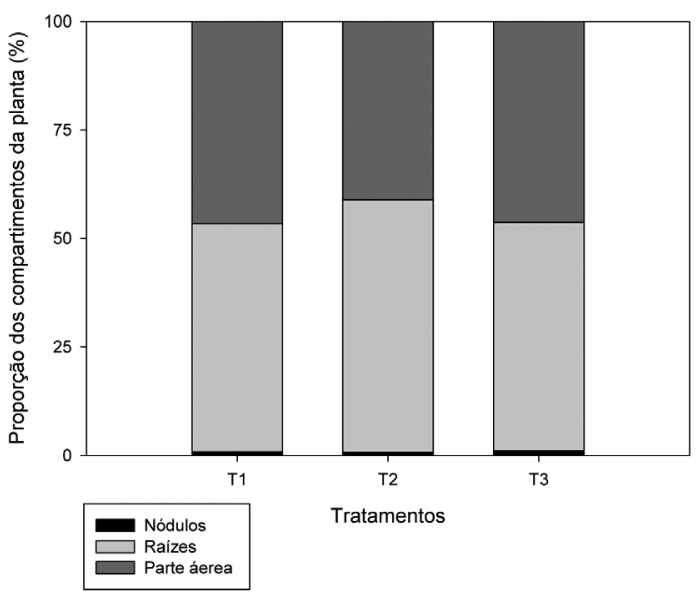

Figura 4 - Proporção dos compartimentos (Nódulos, raízes e parte aérea) de plantas jovens noduladas de $L$. muehlbergianus tratadas durante 20 dias com nitrogênio mineral na forma de nitrato. T1: sem $\mathrm{N}$; $\mathrm{T} 2: \mathrm{NO}_{3}^{-} 7,5$ mM; T3: $\mathrm{NO}_{3}^{-2} 22,5 \mathrm{mM}$.

Figure 4 - Component dry weight of young nodulated plants of $L$. muehlbergianus treated with mineral nitrogen as $\mathrm{NO}_{3}{ }^{-}$for 20 days. T1: no nitrogen; $\mathrm{T}^{2}: \mathrm{NO}_{3}^{-} 7,5 \mathrm{mM}$; $\mathrm{T}^{-} \mathrm{NO}_{3}^{-} 22,5 \mathrm{mM}$

Os valores de ureídeos não diferiram estatisticamente entre os tratamentos em nenhuma das partes da planta (Tab. 1) e em folhas foram similares aos verificados em raízes. $\mathrm{O}$ teor de ureídeos da seiva indicou que a fixação biológica nos nódulos estava plenamente ativa mesmo nos 
Tabela 1 - Teores de aminoácidos totais $\left(\mu \mathrm{mol} \mathrm{g} \mathrm{g}^{-1} \mathrm{MF}^{-1}\right)$ e ureídeos $\left(\mathrm{nmol} \mathrm{g}^{-1} \mathrm{MF}^{-1}\right)$, nitrato $\left(\mu \mathrm{mol} \mathrm{g}^{-1} \mathrm{MF}^{-1}\right)$ e atividade da enzima redutase do nitrato (NRA) ( $\mu \mathrm{mol} \mathrm{NO}_{3}{ }^{-} \mathrm{g}^{-1} \mathrm{MF}^{-1}$ ) em L. muehlbergianus tratadas durante 20 dias com nitrogênio mineral na forma de $\mathrm{NO}_{3}^{-}$. T1: sem N; T2: $\mathrm{NO}_{3}^{-} 7,5 \mathrm{mM}$; $\mathrm{T}^{-}$: $\mathrm{NO}_{3}^{-} 22,5 \mathrm{mM}$.

Table 1 - Total amino acids $\left(\mu \mathrm{mol} \mathrm{g}{ }^{-1} \mathrm{FW}^{-1}\right)$, ureides $\left(\mathrm{nmol} \mathrm{g}{ }^{-1} \mathrm{FW}^{-1}\right)$ and nitrate contents $\left(\mu \mathrm{mol} \mathrm{g}^{-1} \mathrm{FW}^{-1}\right)$ and reductase nitrate activity

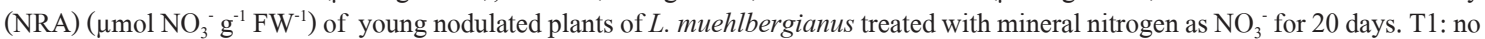
nitrogen; $\mathrm{T} 2: \mathrm{NO}_{3}^{-} 7,5 \mathrm{mM}$; $\mathrm{T} 3: \mathrm{NO}_{3}^{-} 22,5 \mathrm{mM}$.

\begin{tabular}{lccc}
\hline \multicolumn{1}{c}{ Tratamento } & T1 & T2 & T3 \\
\hline Folhas & $3,13^{*} \mathrm{a}^{* *}$ & $2,62 \mathrm{a}$ & $6,85 \mathrm{a}$ \\
Aminoácidos & $584 \mathrm{a}$ & $336 \mathrm{a}$ & $532 \mathrm{a}$ \\
Ureídeos & $8,06 \mathrm{a}$ & $9,43 \mathrm{a}$ & $15,14 \mathrm{a}$ \\
Nitrato & $31,62 \mathrm{a}$ & $179,01 \mathrm{a}$ & $183,53 \mathrm{a}$ \\
NRA & & & $16,45 \mathrm{a}$ \\
Raízes & $13,76 \mathrm{a}$ & $468 \mathrm{a}$ \\
Aminoácidos & $659 \mathrm{a}$ & $19,71 \mathrm{a}$ & $0,31 \mathrm{a}$ \\
Ureídeos & $6,89 \mathrm{a}$ & $993 \mathrm{a}$ & $0,001 \mathrm{a}$ \\
Nitrato & $0,008 \mathrm{a}$ & $9,71 \mathrm{a}$ & $3,12 \mathrm{a}$ \\
NRA & & $0,001 \mathrm{a}$ & $1051 \mathrm{a}$ \\
Seiva & $2,13 \mathrm{a}$ & & $3,08 \mathrm{a}$ \\
Aminoácidos & $695 \mathrm{a}$ & $969 \mathrm{a}$ & \\
Ureídeos & & & \\
\hline
\end{tabular}

* Média de três plantas.

** Médias seguidas pela mesma letra entre os tratamentos não diferem entre si pelo teste de Tukey $(P \leq 0,05)$.

tratamentos em que plantas noduladas foram submetidas ao tratamento com $\mathrm{NO}_{3}{ }^{-}$, pois estes compostos quando transportados pelo xilema podem ser utilizados como parâmetro para a obtenção de estimativas da eficiência da fixação biológica do nitrogênio (McClure \& Israel 1979). Entretanto, esta técnica se limita à determinação desta eficiência apenas em plantas transportadoras de ureídeos, como é o caso da espécie utilizada neste trabalho. O uso de aminoácidos da seiva também já foi utilizado para este fim, principalmente em condições de restrição da fixação biológica por fatores abióticos (Amarante et al. 2006; Justino \& Sodek 2013). Nesta espécie, verificou-se que quantidade razoável de nitrogênio é transportada na forma de ureídeos, uma vez que para cada carbono da constituição dos ureídeos, um nitrogênio é transportado, o que não ocorre com os aminoácidos comumente transportados pela seiva do xilema de leguminosas, como glutamina e asparagina e suas aminas glutamato e aspartato (Amarante et al. 2006). Em C. mucunoides, a adição de $\mathrm{NO}_{3}^{-}$na concentração de $15 \mathrm{mM}$ aumentou a quantidade de ureídeos na seiva do xilema em comparação ao tratamento controle, o que não recebeu nitrogênio mineral. Por outro lado, o tratamento com o amônio não alterou os valores de ureídeos transportados, os quais se mantiveram semelhantes aos verificados em plantas controle (Camargos \& Sodek 2010). Por outro lado, a quantidade de nitrogênio transportada por ureídeos em C. mucunoides é superior à verificada na espécie objeto do presente estudo.

Os aminoácidos desempenharam papel importante no transporte de nitrogênio pelo xilema da espécie estudada neste trabalho (Tab. 1). Foi verificada uma tendência no transporte e acúmulo de aminoácidos em plantas tratadas com $\mathrm{NO}_{3}^{-}$. Foi constatado que L. muehlbergianus acumula aminoácidos preferencialmente no sistema radicular, seguido de folhas. $\mathrm{O}$ aumento no transporte de aminoácidos pelo xilema pode ser indicativo de que uma quantidade adicional de nitrogênio poderia estar sendo metabolizada no sistema radicular e transportada para a parte aérea na forma de aminoácidos. Embora, a 
atividade da enzima redutase do nitrato não tenha sido detectada, os dados apresentados neste trabalho suportam a hipótese de que a espécie L. muehlbergianus poderia utilizar ambas as formas de nitrogênio disponível no meio, $\mathrm{NO}_{3}{ }^{-} \mathrm{e}$ $\mathrm{N}_{2}$, pois foram verificados aumentos nos teores de compostos nitrogenados nos tratamentos em que $\mathrm{O} \mathrm{NO}_{3}^{-}$foi adicionado. Este desempenho sugere que a espécie tem amplitude ecológica no que se refere ao uso do nitrogênio do ambiente, podendo otimizar a entrada de nitrogênio fixado no ambiente, mesmo na presença de nitrogênio na forma mineral.

A atividade da enzima redutase do nitrato aumentou nas folhas de tratamentos que receberam nitrato como fonte de nitrogênio, entretanto, não foi verificada diferença significativa entre os tratamentos (Tab. 1). A presença do nitrato aumentou mais de sete vezes em folhas de plantas de Calopogonium mucunoides tratadas com $15 \mathrm{mM}$ de nitrato (Camargos \& Sodek 2010). No mesmo trabalho, o aumento da atividade enzimática foi acompanhado por aumento dos teores de nitrato nos tecidos foliares, tendência encontrada em nossos resultados. Ainda, a atividade dobrou em raízes tratadas com nitrato, resultado inferior ao verificado na parte aérea. A atividade detectada em L. muehlbergianus tratada com nitrato não aumentou e os valores foram significativamente baixos, o que poderia indicar que a atividade de redução do nitrato ocorre preferencialmente nas folhas. Os valores encontrados em folhas de L. muehlbergianus tratada com nitrato foram maiores do que os verificados em C. mucunoides, entretanto, os teores de nitrato foliar em $L$. muehlbergianus foram menores. De acordo com dados da literatura, a intensidade da atividade da redutase do nitrato depende do grupo de sucessão e da interação das espécies, bem como das alterações dos fatores ambientais, como disponibilidade de luz e precipitação (Aidar et al. 2003). De fato, estes pesquisadores encontraram atividades bastante superiores em espécies pioneiras quando comparadas com espécies sucessionais iniciais e tardias em estudo realizado na Mata Atlântica. Neste mesmo trabalho, os autores encontraram atividades da redutase do nitrato relativamente altas em leguminosas (Inga marginata Willd., Piptadenia gonoacantha (Mart.) J.F. Macbr. e Zchizolobium parahyba (Vell.) S.F. Blake) enquanto que Barreto et al. (2007) trabalhando com plantas jovens de Aniba rosaeodora (Ducke) (com quatro meses de idade), uma espécie da família Lauraceae, observaram atividades muito baixas quando tratadas com nitrato em casade-vegetação. Neste último, foi verificado que a atividade enzimática foi estimulada quando doses crescentes de nitrato foram utilizadas, comportamento similar ao observado no presente trabalho, realizado com L. muehlbergianus. Este resultado é esperado, uma vez que a atividade desta enzima, entre outros fatores, é induzida pelo substrato.

\section{Conclusão}

Nas condições em que o experimento foi realizado, a fixação biológica do nitrogênio em L. muehlbergianus não é aparentemente afetada pela presença de nitrogênio mineral $\left(\mathrm{NO}_{3}{ }^{-}\right)$no meio de cultivo. Entretanto, a disponibilidade de $\mathrm{NO}_{3}^{-}$não aumenta relevantemente o acúmulo de biomassa total nesta espécie, no tempo em que o experimento foi realizado. Ademais, os dados suportam a hipótese de que a espécie poderia utilizar tanto nitrogênio mineral quanto nitrogênio molecular no ambiente natural, potencializando a entrada de nitrogênio fixado no ambiente, favorecendo outras famílias botânicas que não tem a capacidade de se associar simbioticamente a bactérias fixadoras de nitrogênio.

\section{Agradecimentos}

Os autores agradecem o apoio financeiro ao CNPq (processos 470160/2003-9 e 473594/20072) e à FAPESP (processo 2010/05299-6); e Vitor Moreira agradece a bolsa fornecida pelo (PIBIC/ CNPq/UFMS-Brasil).

\section{Referências}

Aidar, M.P.M.; Schmidt, S.; Moss, G.; Stewart, G.R. \& Joly, C.A. 2003. Nitrogen use strategies of neotropical rainforest trees in threatened Atlantic Forest. Plant, Cell and Environment 26: 389-399.

Amarante, L. \& Sodek, L. 2006. Waterlogging effect on xylem sap glutamine of nodulated soybean. Biologia Plantarum 50:405-410.

Amarante L.; Lima, J.D. \& Sodek L. 2006. Growth and stress conditions cause similar changes in xylem amino acids for different legume species. Environmental and Experimental Botany 58: 123-129.

Barreto, D.C.S.; Gonçalves, J.F.C.; Santos-Junior, U.M.; Fernandes, A.V.; Bariane, A. \& Sampaio, P.T.B. 2007. Biomass accumulation, photochemical 
efficiency of photosystem II, nutrient contents and nitrate reductase activity in young rosewood plants (Aniba rosaeodora Ducke) submitted to different $\mathrm{NO}_{3}{ }^{-}: \mathrm{NH}_{4}{ }^{+}$ratios. Acta Amazonica 37: 533-542.

Betts, J.H. \& Herridge, D.F. 1987. Isolation of soybean lines capable of nodulation and nitrogen fixation under high levels of nitrate supply. Crop Science 27: 1156-1161.

Bieleski, R.L. \& Turner, N.A. 1966. Separation and estimation of amino acids in crude plant extracts by thin-layer electrophoresis and chromatography. Analytical Biochemistry 17: 278-293.

Camargos, L.S. \& Sodek, L. 2010. Nodule growth and nitrogen fixation of Calopogonium mucunoides L. show low sensitivity to nitrate. Symbiosis 51: 167-174.

Cancian, M.A.E. 1999. Efeito do sombreamento e da temperatura do substrato na nodulação e desenvolvimento inicial de Anadenanthera columbrina (Vell.) Brenan, Lonchocarpus muehlbergianus Hass e Ciclolobium vecchi A. Samp. Hex Hoene. Tese de Doutorado. Universidade Estadual Paulista, Rio Claro. 130p.

Cancian, M.A.E. \& Cordeiro, L. 1998. Efeito do sombreamento no crescimento inicial de Lonchocarpus muehlbergianus Hassl. Acta Botanica Brasilica 12: 367-372.

Carroll, B.J.; McNeil, D.L. \& Gresshoff, P.M. 1985a. Isolation and properties of soybean [Glycine max (L.) Merr.] mutants that nodulate in the presence of high nitrate concentrations. Proceeding of the National Academy of Sciences 82: 4162-4166.

Carroll, B.J.; McNeil, D.L. \& Gresshoff, P.M. 1985 b. A supernodulation and nitrate-tolerant symbiotic (nts) soybean mutant. Plant Physiology 78: 34-40.

Michaelson-Yeates, T.P.T.; Macduff, J.H.; Abberton, M.T. \& Raistrick, N. 1998. Characterization of novel inbred lines of white clover (Trifolium repens L.). II. Variation in $\mathrm{N}_{2}$ fixation, $\mathrm{NO}_{3}{ }^{-}$uptake and their interactions. Euphytica 103: 45-54.

Dan, T. H. \& Brix, H. 2009. Growth responses of the perennial legume Sesbania sesban to $\mathrm{NH}_{3}$ and $\mathrm{NO}_{3}$ nutrition and effects on root nodulation. Aquatic Botany 91: 238-244.

Forrester D.I.; Bauhus J.; Cowie, A.L.; Partap K. \& Vanclay, J.K. 2006. Mixed-species plantations of eucalyptus with nitrogen-fixing trees. Forest Ecology and Management 233: 211-230.

Glyan'ko, A.K.; Vasil'eva, G.G.; Mitanova, N.B. \& Ishchenko, A.A. 2009. The influence of mineral mitrogen on legume-rhizobium symbiosis. Biology Bulletin 36: 302-312.

Groten, K.; Vanacker, H.; Dutilleul, C.; Bastian, F.; Bernard, S.; Carzaniga, R. \& Foyer C.H. 2005. The roles of redox processes in pea nodule development and senescence. Plant, Cell and Environment 28: 1293-1304.

Hardarson, G. \& Atkins, C. 2003. Optimizing biological $\mathrm{N}_{2}$ fixation by legumes in farming systems. Plant and Soil 252: 41-54.

Hardarson, G; Danso, S.K.A.; Zapata, F. \& Reichardt, K. 1991. Measurements of nitrogen fixation in fababean at different $\mathrm{N}$ fertilizer rates using $15 \mathrm{~N}$ isotope dilution and 'A-value' methods. Plant and Soil 131: 161-168.

Herridge, D.F. \& Betts, J.H. 1985. Nitrate tolerance in soybean: variation between genotypes. In: Evans, H.J.; Bottomley, P.J. \& Newton, W.E. (eds.) Nitrogen fixation research progress. Martinus Nijhoff, Dordrecht. P. 32.

Herridge, D.F. \& Betts, J.H. 1988. Field evaluation of soybean genotypes selected for enhanced capacity to nodulate and fix nitrogen in the presence of nitrate. Plant and Soil 110: 129-135.

Herridge, D. \& Rose, I. 2000. Breeding for enhanced nitrogen fixation in crop legumes. Field Crops Research 65: 229-248.

Hoagland, D.R \& Arnon, D.I. 1938. The water-culture method for growing plants without soil. University of California Agricultural Experimental Station, Berkeley. 39p.

Instituto Florestal de São Paulo - IF. Secretaria do Meio Ambiente - SMA. Instituto de Pesquisas Ecológicas - IPÊ. 2003. Plano de manejo do Parque Estadual do Morro do Diabo: espécies vegetais registradas nas diferentes fitofisionomias do PEMD. SMA, São Paulo. 312p.

Jacobsen, E. \& Feenstra, W.J. 1984. A new pea mutant with eficient nodulation in the presence of nitrate. Plant Science Letters 33: 337-344.

Justino, G.C. \& Sodek, L. 2013. Recovery of nitrogen fixation after short-term flooding of the nodulated root system of soybean. Journal of Plant Physiology 170: 235-241.

Lorenzi, H. 2000. Árvores brasileiras: manual de identificação e cultivo de plantas arbóreas nativas do Brasil. Vol. 1. $3^{\mathrm{a}}$ ed. Instituto Plantarum de Estudos da Flora, Nova Odessa. 352p.

McClure, P.R. \& Israel, D.W. 1979. Transport of nitrogen in the xylem of soybean plants. Plant Physiology 64: 411-416.

Dakora, F.D. 1998. Nodule function in symbiotic bambara groundnut (Vigna subterranea L.) and Kersting's bean (Macrotyloma geocarpum L.) is tolerant of nitrate in root medium. Annals of Botany 82: 687-690.

Nichols, J.D. \& Carpenter, F.N. 2006. Interplanting Inga edulis yields nitrogen benefits to Terminalia Amazonia. Forest Ecology and Management 233: 344-351.

Omena-Garcia, R.P.; Justino, G.C.; Sodek, L. \& Gonçalves, J.F.C. 2011. Mineral nitrogen affects 
nodulation and amino acids xylem transport in the Amazonian legume Inga edulis Mart. International Journal of Plant Physiology and Biochesmistry 3: 215-218.

Park, S.J. \& Buttery, B.R. 1988. Nodulation mutants of white bean (Phaseolus vulgaris L.) induced by ethyl-methane sulphonate. Canadian Journal of Plant Science 68: 199-202.

Park, S.J. \& Buttery, B.R. 1989. Identification and characterization of common bean (Phaseolus vulgaris L.) lines well nodulated in the presence of high nitrate. Plant and Soil 119: 237-244.

Radin, J.W. 1973. "In vivo" assay of nitrate reductase in cotton leaf discs. Plant Physiology 51: 332-336.

Scholander, P.F.; Bradstreet, E.D.; Hemmingsen, E.A. \& Hammel, H.T. 1965. Sap pressure in vascular plants:
Negative hydrostatic pressure can be measured in plants. Science 148: 39-46.

Sodek, L. \& Silva, D.M. 1996. Nitrate inhibits soybean nodulation and nodule activity when applied to root regions distant from the nodulation sites. Revista Brasileira de Fisiologia Vegetal 8: 187-191.

Unkovich, M.; Herridge, D.; Peoples, M.; Cadisch, G.; Boddey, B.; Giller, K.; Alves, B. \& Chalk, P. 2008. Measuring plant-associated nitrogen fixation in agricultural systems. No. 136. ACIAR Monograph, Canberra 258p.

Vogels, G.D. \& Van Der Drift, C. 1970. Differential analysis of glycolate derivatives. Analytical Biochemistry 33: 143-157.

Yemm, E.W. \& Cocking, E.C. 1955. The determination of amino acids with ninhydrin. Analyst 80: 209-213. 